Originalien

Monatsschr Kinderheilkd

https://doi.org/10.1007/s00112-021-01198-6

Angenommen: 22. März 2021

() Der/die Autor(en) 2021

\section{Redaktion}

Berthold Koletzko, München

Thomas Lücke, Bochum

Ertan Mayatepek, Düsseldorf

Norbert Wagner, Aachen

Stefan Wirth, Wuppertal

Fred Zepp, Mainz
E. Verjans ${ }^{1} \cdot$ U. Fabry ${ }^{2} \cdot$ N. Wagner ${ }^{1}$

' Klinik für Kinder- und Jugendmedizin, Medizinische Fakultät, Universitätsklinikum Aachen, RWTH Aachen, Aachen, Deutschland

${ }^{2}$ Medizinisches Controlling, Medizinische Fakultät, Universitätsklinikum Aachen, RWTH Aachen, Aachen, Deutschland

\title{
Deutlich erhöhte Sterberate bei Kindern von Eltern mit Konsum von illegalen, harten Drogen
}

\section{Ergebnisse einer monozentrischen Studie über sechs Jahre}

\section{Einleitung \\ Situation in Deutschland}

Deutschlandweit leben aktuell über 3 Mio. Kinder in einem Haushalt mit mindestens einem suchtkranken Elternteil. Immerhin etwa 60.000 Kinder stammen aus Familien, in denen ein oder beide Elternteil/e einen Drogenabusus betreiben, wobei Drogen hier als rauscherzeugende Substanzen mit Ausnahme von Alkohol und Medikamenten definiert werden [8]. Die Dunkelziffer wird in beiden Fällen erheblich höher angenommen [5].

\section{Situation in Aachen}

In der Stadt Aachen wird die Zahl der von harten, illegalen Drogen (definiert als Heroin, Crack, Methamphetamin, Kokain etc., kein Cannabis) Abhängigen mit 1500-2000 angegeben (Auskunft der Stadt Aachen). Nach Recherchen der Autoren leben ca. 6700 Kinder in einer Familie mit mindestens einem suchtkranken Elternteil (Alkohol, Medikamente, Drogen aller Art). Hieraus ergibt sich eine kalkulierte Zahl von 100-200 Kindern aus Familien mit hartem Drogenkonsum (prozentual abgeleitet aus den bundesweiten Zahlen), wobei der genaue Anteil unklar bleibt.

Zum Schutz der Kinder werden vielfach schon Neugeborene von drogenab- hängigen Eltern direkt nach der Geburt in Obhut genommen und an Pflegefamilien vermittelt bzw. durchlaufen das Adoptionsverfahren. In selteneren Fällen verbleiben die Kinder mit dafür vorgesehenen Hilfesystemen bei den leiblichen Eltern. Bei unbekanntem Drogenkonsum der Eltern können unterstützende Maßnahmen nicht installiert werden. Insgesamt kommt es trotz weitreichenden, verfügbaren Hilfesystemen und hohem Ressourceneinsatz immer wieder zu einer Gefährdung des Kindeswohls mit teils dramatischem Ausgang.

\section{Fokus Eltern-Kind-Verhältnis in drogenbelasteten Familien}

Eine Abhängigkeit von harten, illegalen Drogen geht meist mit einer Partnerschaft innerhalb der Konsumentengruppe einher, sodass sowohl Mutter als auch Vater häufig an einer Abhängigkeitserkrankung leiden. Dies steht im Gegensatz zur Alkoholsucht, bei der meist nur ein Elternteil betroffen ist [11]. Verbleiben die Kinder in den entsprechenden Familien, erleben sie häufig die typischen Bedingungen der Drogensubkultur, verbunden mit Beschaffungskriminalität der Eltern, wie Prostitution, und Strafverfolgung [2] sowie Fremdunterbringung oder Inobhutnahmen. Hierbei sind v. a. Kleinkinder als hochvulnerabel anzusehen, da in dieser jungen Altersklasse die individuelle Persönlichkeitsentwicklung einen hohen Stellenwert einnimmt. Die Kinder sind jedoch auf der anderen Seite noch verletzlich und auf die Zuwendung der Eltern angewiesen. Daher ergibt sich gerade hier bei elterlichem Drogenmissbrauch ein erhöhtes Risiko für schwere Kindesmisshandlung mit gravierenden Spätfolgen $[6,9]$.

\section{》) Elterlicher Drogenmissbrauch steigert das Risiko für schwere Kindesmisshandlungen}

Schon lange ist bekannt, dass Kinder aus suchtbelasteten Familien mit höherer Wahrscheinlichkeit Opfer oder Zeuge von häuslicher Gewalt werden [12]. Dies führt neben langfristigen kognitiven/motorischen und sozial-emotionalen Defiziten der Kinder [13, 15] teils auch zu Situationen mit akutem Gefährdungspotenzial. Diese Kinder bleiben meist nicht nur unbeteiligte Dritte, sondern sind selbst in Konflikte involviert oder unterliegen körperlicher Gewalt durch ein Elternteil [1, 3].

Insgesamt kann somit die Aussage, dass Kinder aus Familien mit elterlichem Drogenabusus einer besonderen Gefahr ausgesetzt sind, zunächst trivial erscheinen. Einzelfallberichte von dramatischen Schicksalen, bei denen Kinder teils zu Tode kommen, sind immer wieder in der Tagespresse zu finden. Ein wissenschaftlicher Beleg für diesen Sachverhalt mithilfe 


\section{Kasuistik 1}

Kind

Eltern

Akutes Ereignis

Rechtsmedizinische Untersuchung/ nachfolgende Informationen

Unklarheiten

Form der Kindeswohlgefährdung/ strafrechtliche Einstufung

Kasuistik 2

Kind

Eltern

Akutes Ereignis

Rechtsmedizinische Untersuchung/ nachfolgende Informationen

Unklarheiten

Form der Kindeswohlgefährdung/ strafrechtliche Einstufung

\section{Kasuistik 3}

Kind

Eltern

Akutes Ereignis

Rechtsmedizinische Untersuchung/ nachfolgende Informationen

Unklarheiten

Form der Kindeswohlgefährdung/ strafrechtliche Einstufung
2 Jahre altes Mädchen, ehem. Frühgeborenes $25+1$ SSW, $860 \mathrm{~g}$, trotz Unreife relativ blander postnataler Verlauf ohne neonatales Entzugssyndrom, im Verlauf rezidivierende pulmonale Infektionen

Alleinerziehende Mutter, 29 Jahre, Z. n. Opiat- und Kokainabusus, Methadonsubstitution seit 2007, galt als zuverlässig, „,Takehome"-Methadon-Dosen durch Substitutionspraxis, postnatal zunächst Entlassung mit sozialpädagogischer Familienhebamme (SPFH), dann Aufnahme in eine Mutter-Kind-Einrichtung bis einen Monat vor dem Ereignis

Akute Bronchitis des Kindes, nach dem Mittagsschlaf lebloses Kind im Bett durch Mutter aufgefunden, Laienreanimation, Reanimation durch Notarzt fortgesetzt, fast durchgehende Asystolie, bei Eintreffen in der Klinik lichtstarre Pupillen und rechts entrundete Pupille, pH-Wert 6,6, Lactatkonzentration $16 \mathrm{mmol} / \mathrm{l}$, keine äußeren Verletzungszeichen, Beendigung der Reanimationsmaßnahmen; einzige Auffälligkeit am Einsatzort: hämatinhaltiger Magenrest des Kindes

Hohe Methadonspiegel im Blut des Kindes als Zeichen einer letalen Intoxikation, Tod als direkte Folge einer oralen Einnahme

Bis zuletzt nicht geklärt, ob Mutter die "Take-home“-Dosis in einer Überforderungssituation dem Kind selbst verabreicht hat (Annahme der Richter im Prozess), oder ob die kindersichere Medikamentendose durch das Kind akzidentiell geöffnet wurde Körperliche Misshandlung, ggf. auch Vernachlässigung der Aufsicht; Körperverletzung mit Todesfolge (§ 227 StGB), ggf. auch fahrlässige Tötung (§ 222 StGB) oder Totschlag (§ 212 StGB)

\section{3,5 Jahre alter, zuvor gesunder Junge}

Alleinerziehende Mutter, 29 Jahre, seit 2 Wochen neuer Partner, 30 Jahre, Aufenthalt im elterlichen Haus des Partners, Eltern des Partners waren zur Zeit des Ereignisses verreist

Das Kind wurde leblos am Ende einer 16-stufigen Treppe aufgefunden, Laienreanimation durch Mutter im Garten, Notarzt findet lebloses Kind, bei Eintreffen in der Klinik Asystolie, pH-Wert 6,6 und Lactatkonzentration $19 \mathrm{mmol} / \mathrm{l}$, ausgeprägte Kopfverletzungen mit schmalem bifrontalem und parafalzinem Subduralhämatom, dezente rechtsseitige parietale Subarachnoidalblutung, leichte generalisierte Hirnschwellung, jedoch mit basal abgrenzbaren Zisternen und erhaltener Mark-RindenDifferenzierung, Monokelhämatom am linken Auge, Mittelgesichtshämatome unterschiedlichen Alters, okzipitales Galeahämatom, Hämatome an beiden Ohrmuscheln, Schürfverletzungen am Hals, großflächiges Hämatom im Bereich der Spina iliaca; zudem V.a. Aspirationsereignis mit nachfolgender Hypoxämie (exemplarische Fotodokumentation in • Abb. 1) Speed-Konsum der Mutter mit ihrem neuen Partner am Vorabend des Ereignisses, darunter Verlust der Zeitwahrnehmung und Erinnerungslücken über einen Zeitraum von fast $24 \mathrm{~h}$, Freund der Mutter nimmt sich selbst am Ende einer spektakulären Verfolgungsjagd mit der Polizei das Leben

Bis zuletzt unklar, wer die körperliche Misshandlung des Jungen begangen hat, oder ob ein reines Unfallgeschehen bei unterlassener Aufsichtspflicht vorlag

Körperliche Misshandlung; vorsätzliche Körperverletzung mit Todesfolge (§ 227 StGB), ggf. Totschlag durch Unterlassung (§§ 212 und 13, Absatz 1 StGB)

Weibliches Frühgeborenes, geschätzt 28./29. SSW, keine Dysmorphiezeichen

Beide heroinabhängig, zusammenlebend in einem Haushalt, Mutter: 29 Jahre, weitere Angaben zum Vater nicht vorliegend Spontanpartus in der eigenen Wohnung, Verständigung des Notarztes, findet totes Kind ohne Lebenszeichen im Ausspültrakt der Toilette, kopfüber unter Wasser

Leichnam wirkte wie ein lebensfrisches Frühgeborenes von 1200-1400 g, keine Zeichen eines intrauterinen Fruchttods/ Spätaborts

Unklar, wer das Kind in die Toilette überführt hat

Körperliche Misshandlung; Totschlag im Sinne eines Neonatizids (§§ 212-213 StGB) 
Monatsschr Kinderheilkd https://doi.org/10.1007/s00112-021-01198-6

(c) Der/die Autor(en) 2021

\section{E. Verjans $\cdot$ U. Fabry $\cdot$ N. Wagner}

\section{Deutlich erhöhte Sterberate bei Kindern von Eltern mit Konsum von illegalen, harten Drogen. Ergebnisse einer monozentrischen Studie über sechs Jahre}

\section{Zusammenfassung}

Hintergrund. In Deutschland leben mindestens 60.000 Kinder in drogenbelasteten Familien und damit im Milieu der Drogensubkultur, das oft mit Gewalt, Beschaffungskriminalität und Prostitution verbunden ist. Diese Kinder weisen eine höhere Wahrscheinlichkeit auf, Opfer oder Zeuge häuslicher Gewalt zu werden. In den letzten 30 Jahren sind jedoch keine systematischen Studien erfolgt, die kindliche Todesfälle bei elterlichem illegalen, harten Drogenabusus analysierten.

Ziel der Arbeit. Untersucht wurde, inwieweit der Gebrauch illegaler, harter Drogen durch die Eltern mit dem Tod oder schwerster Schädigung des Kindes assoziiert ist.

\author{
Material und Methoden. In der retrospektiven \\ Kohortenstudie wurden vom 01.08.2013 bis \\ zum 01.08.2019 alle Neuaufnahmen in der \\ Klinik für Kinder- und Jugendmedizin des \\ Uniklinikums Aachens analysiert. Patienten \\ (Alter 0 bis 18 Jahre), bei denen mindestens \\ ein Elternteil einen Drogenabusus (außer \\ Alkohol und Medikamenten) aufwies und \\ dieser vermutlich unmittelbar zur akuten \\ Lebensbedrohung des Kindes geführt \\ bzw. dessen Tod verursacht hatte, wurden \\ identifiziert. \\ Ergebnisse. Unter 29.141 stationären \\ Aufnahmen entsprachen 5 Fälle den \\ Einschlusskriterien, hiervon 3 Fälle mit \\ Todesfolge, ein Fall mit schwerer psychomo- \\ torischer Beeinträchtigung nach der Tat unter
}

Drogeneinfluss und ein Fall, bei dem die akute Gefährdung abgewendet werden konnte. Die Sterbewahrscheinlichkeit für Kinder von Eltern, die illegale, harte Drogen konsumieren, war 10-fach höher als in der Kontrollgruppe. Schlussfolgerung. Der Gebrauch illegaler, harter Drogen durch die Eltern stellt v.a. in der Altersklasse von 0 bis 5 Jahren einen relevanten Risikofaktor der Kindeswohlgefährdung mit schwersten Folgen bis zum Tod dar und führt zu einer deutlich erhöhten Todeswahrscheinlichkeit.

Schlüsselwörter Kindeswohl · Eltern-Kind-Beziehung · Substanzgebundene Störungen · Häusliche Gewalt $\cdot$ Totschlag

\section{Markedly increased mortality rate in children of parents on illegal hard drugs. Results of a 6-year monocentric study}

\section{Abstract}

Background. At least 60,000 children in Germany live in a domestic environment of illegal drug abuse, often combined with violence, drug-related crime and prostitution. These children are at high risk of becoming victims or witnesses of domestic violence; however, no systematic studies have been published in the last 30 years reviewing fatalities in connection with parental abuse of illegal hard drugs.

Objective. To investigate to what extent parental consumption of illegal hard drugs is associated with death or severe bodily harm to the child.

Material and methods. A retrospective, unicentric cohort study at the University
Hospital of Aachen was conducted over a period of 72 months investigating the frequency of fatalities or severe bodily harm to children in connection with parental use of illegal hard drugs in Aachen. All patients (age 0-18 years) admitted to the department of pediatrics at the University Hospital of Aachen, where parental drug abuse (apart from alcohol and medication) had most probably had lifethreatening or fatal consequences for the child were identified.

Results. Of the total cohort of 29,141 inpatient admissions reviewed 5 met the inclusion criteria. In three cases parental drug abuse led to the child's death, in one case the psychomotor development of the child was dramatically delayed and in one case a fatality was avoided by taking the child out of danger. The probability of death was tenfold higher in children whose parents were on illegal hard drugs than in the control group. Conclusion. Parental consumption of illegal hard drugs is a relevant risk factor for endangerment of child welfare with severe and even fatal consequences, particularly in the age group of $0-5$ years and leads to a markedly increased probability of death.

\section{Keywords}

Child welfare - Parent-child relations . Substance-related disorders - Domestic violence $\cdot$ Manslaughter systematischer Analysen von Todesfällen bzw. schwersten Schädigungen bei elterlichem Drogenabusus ist jedoch in der Literatur - nach den Recherchen der Autoren - in den letzten Jahrzehnten nicht zu finden. Daher zielt die vorliegende Studie darauf ab, exemplarisch eine erste quantitative Analyse der Situation in einer deutschen Großstadt vorzustellen.

\section{Material und Methoden}

In einer retrospektiven unizentrischen Kohortenstudie über einen Beobachtungszeitraum von 72 Monaten (01.08. 2013 bis 01.08.2019) wurde untersucht, mit welcher Häufigkeit Fälle von Kindeswohlgefährdung mit akuter Lebensbedrohung bzw. tödlichem Ausgang unter Drogenkonsum der Eltern aufgetreten sind. Hierbei wurden retrospektiv die Neuaufnahmen in der Klinik für
Kinder- und Jugendmedizin des Uniklinikums (UK) Aachen während des oben genannten Zeitraums betrachtet. Es erfolgte eine Selektion aller Patienten (Alter 0 bis 18 Jahre), bei denen mindestens ein Elternteil einen Abusus von harten Drogen (Definition s. oben) aufwies und dieser unmittelbar zu einer akuten Lebensbedrohung des Kindes bzw. sogar zum Tod geführt hatte. Es wurden sowohl Fälle eingeschlossen, bei denen der Drogenkonsum bereits di- 

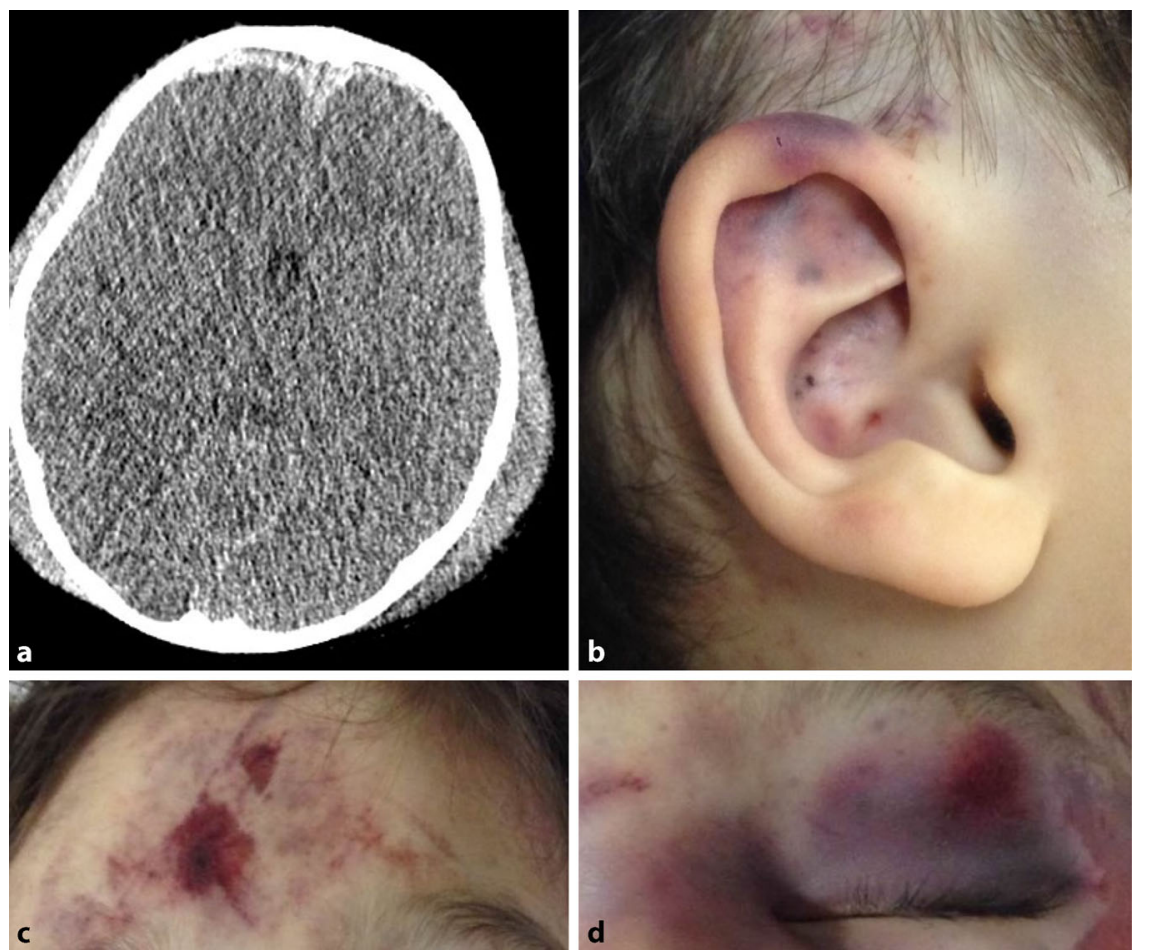

Abb. 1 A Fotodokumentation zu Kasuistik 2. a Kranielle Computertomographie am Aufnahmetag mit bifrontalem Subduralhämatom und beidseitiger deutlicher Weichteilschwellung, b Hämatome im Bereich der rechten Ohrmuschel, $\mathrm{c}$ Hämatome und Abschürfungen im Bereich der Stirn, $\mathbf{d}$ Hämatom des linken Oberlids

rekt bei stationärer Aufnahme bekannt war, als auch solche, bei denen sich der Drogenkonsum als unmittelbarerer Hauptfaktor für die Kindeswohlgefährdung in einem späteren strafrechtlichen Prozess herausgestellt hat.

\section{Ergebnisse}

Im genannten Zeitraum erfolgten 29.141 stationäre Aufnahmen in der Klinik für Kinder- und Jugendmedizin des UK Aachen, wobei folgende 5 Fälle den oben genannten Kriterien zugeordnet werden konnten:

- 3 Fälle mit Todesfolge (• Tab. 1,

Kasuistiken 1-3; exemplarische

Fotodokumentation der Kasuistik 2

in $\bullet$ Abb. 1),

- ein Fall mit schwerer psychomotorischer Beeinträchtigung (•Tab. 2, Kasuistik 4),

- ein Fall mit abgewendeter Gefährdung (『Tab. 2, Kasuistik 5).

Die in - Tab. 1 dargestellten Todesfälle entsprechen 3,6\% aller Todesfälle (3 von insgesamt 82 Fällen), die im Beobach-
Freund der Mutter (Kasuistik 2), der Körperverletzung beschuldigt.

Nach dem Bürgerlichen Gesetzbuch $\$ 1666$ liegt eine Kindeswohlgefährdung vor, wenn ...das körperliche, geistige oder seelische Wohl des Kindes oder sein Vermögen gefährdet und ... die Eltern nicht gewillt oder nicht in der Lage sind, die Gefahr abzuwenden. Hierbei werden folgende Formen der Kindeswohlgefährdung unterschieden (u.a. nach Leeb [10]):

- Vernachlässigung der Grundversorgung des Kindes,

- Vernachlässigung der Fürsorge und Aufsicht,

- Vernachlässigung der seelischen und geistigen Entwicklung des Kindes,

- körperliche Misshandlung,

- seelische Kindesmisshandlung,

- Missbrauch der elterlichen Sorge.

Die Einschätzungen der Autoren bezüglich der Tatbestände bzw. die strafrechtlichen Beurteilung sind in den - Tab. 1 und 2 ergänzend zu den jeweiligen Kasuistiken aufgeführt. Anhand der schwerwiegenden Strafmaße, die sich aus den einzelnen Straftatbeständen ergeben, kann noch einmal hervorgehoben werden, dass harter Drogenkonsum der Eltern das Risiko für eine schwerste Beeinträchtigung bzw. den Tod der Kinder deutlich erhöht.

Drogen. In einem Fall war nur die Drogenabhängigkeit bekannt, jedoch nicht die genaue Substanz (kein Alkohol, keine Medikamente, Kasuistik 4), die konsumiert wurde.

Die 3 Todesfälle lassen sich einteilen in:

- direkte Schädigung durch rauscherzeugende Substanzen (Kasuistik 1),

- Schädigung durch ein verändertes Verhalten der Eltern unter Drogeneinfluss in Form von Ablehnung (Kasuistik 3) und körperlicher Gewalt (Kasuistik 2, • Abb. 1).

Bei der Betrachtung, wer den Kindern akuten Schaden zugefügt oder durch Ablehnung diese Situation herbeigeführt hatte, zeigt sich, dass in 4 Fällen ein Elternteil oder beide Elternteile beteiligt waren. In einem Fall wurde eine nahe Kontaktperson, nämlich der neue
) Die Mortalitätsrate für 0- bis 5-Jährige aus Familien mit hartem Drogenabusus ist 10-fach erhöht

In der Klinik für Kinder- und Jugendmedizin des UK Aachen wurden somit im Studienzeitraum 3 Todesfälle und ein Kind mit schweren Langzeitschäden vor dem Hintergrund eines elterlichen Drogenkonsums identifiziert. Diese Zahlen erscheinen zunächst nicht gravierend, prozentual betrachtet handelt es sich jedoch um 3,6\% aller kinderklinisch dokumentierten Todesfälle im Studienzeitraum. Um die Auswertung in ihrer Bedeutung zu untermauern, wurde die Wahrscheinlichkeit eines Todesfalls in der Population der Kleinkinder von dro- 
Tab. 2 Kurzdarstellung der Kasuistiken ohne Todesfolgen

\begin{tabular}{|c|c|}
\hline $\begin{array}{l}\text { Erhobene Parame- } \\
\text { ter }\end{array}$ & Beschreibung \\
\hline \multicolumn{2}{|l|}{ Kasuistik 4} \\
\hline Kind & 6 Wochen alter, bisher gesunder männlicher Säugling, „Schreikind“ \\
\hline Eltern & $\begin{array}{l}\text { Leben zusammen in einem Haushalt, Mutter } 19 \text { Jahre, Alter des Vaters nicht } \\
\text { genau bekannt }\end{array}$ \\
\hline Akutes Ereignis & $\begin{array}{l}\text { Kind abends zum Schlafen hingelegt, dann wimmernde Geräusche aus } \\
\text { dem Kinderzimmer gehört, Kind sei nicht gut erweckbar gewesen, Kind } \\
\text { bei Eintreffen im Klinikum hypoton, blass, schwer erweckbar, fokaler, dann } \\
\text { sekundär generalisierter Krampfanfall, schwer durchbrechbar, beidseitige } \\
\text { Subduralhämatome bei V.a. Brückenvenenabriss, im Verlauf große Hygro- } \\
\text { me beidseits, retinale Blutungen beidseits, hypoxischer Hirnschaden mit } \\
\text { globaler Hirnvolumenminderung }\end{array}$ \\
\hline $\begin{array}{l}\text { Rechtsmedizinische } \\
\text { Untersuchung/ } \\
\text { nachfolgende Infor- } \\
\text { mationen }\end{array}$ & $\begin{array}{l}\text { Drogenabusus des Vaters, durch ihn beigebrachtes Schütteltrauma einige } \\
\text { Tage vor der Einlieferung }\end{array}$ \\
\hline Unklarheiten & Unklar, welche Drogen konsumiert wurden \\
\hline $\begin{array}{l}\text { Form der Kindes- } \\
\text { wohlgefährdung/ } \\
\text { strafrechtliche Ein- } \\
\text { stufung }\end{array}$ & $\begin{array}{l}\text { Körperliche Misshandlung; schwere Körperverletzung (§ } 226 \text { StGB Absatz } 1 \\
\text { Nr. 1-3) }\end{array}$ \\
\hline \multicolumn{2}{|l|}{ Kasuistik 5} \\
\hline Kind & Reifes weibliches AGA-Neugeborenes, $38+4$ SSW \\
\hline Eltern & $\begin{array}{l}\text { Mutter: } 21 \text { Jahre, bekannter Cannabis- und Kokainabusus, Vater: Heroinab- } \\
\text { hängigkeit, Alter des Vaters nicht genau bekannt }\end{array}$ \\
\hline Akutes Ereignis & $\begin{array}{l}\text { Spontanpartus als Hausgeburt, Nabelschnur wurde durchgerissen, nach } \\
2 \text { Tagen wurde das Kind in einer dreckigen, unbewohnbaren, vermüllten } \\
\text { Wohnung gefunden, Eltern unter Drogeneinfluss, keine direkte Schädigung } \\
\text { des Kindes, kein ausgeprägtes neonatales Abstinenzsyndrom }\end{array}$ \\
\hline $\begin{array}{l}\text { Rechtsmedizinische } \\
\text { Untersuchung/ } \\
\text { nachfolgende Infor- } \\
\text { mationen }\end{array}$ & $\begin{array}{l}\text { Nachweis von Kokain im Urin des Kindes, wahrscheinlich durch maternalen, } \\
\text { präpartalen Konsum, Kind sollte durch das Jugendamt direkt postnatal } \\
\text { in der Geburtsklinik in Obhut genommen werden, daher wahrscheinlich } \\
\text { Hausgeburt }\end{array}$ \\
\hline $\begin{array}{l}\text { Form der Kindes- } \\
\text { wohlgefährdung/ } \\
\text { strafrechtliche Ein- } \\
\text { stufung }\end{array}$ & $\begin{array}{l}\text { Vernachlässigung der Grundversorgung des Kindes und Vernachlässigung } \\
\text { der Fürsorge und Aufsicht; Körperverletzung durch Unterlassen (§§ } 223 \\
\text { und } 13 \text { StGB) }\end{array}$ \\
\hline enrepriato fo & stational age" \\
\hline
\end{tabular}

genabhängigen Eltern am Beispiel der Stadt Aachen kalkuliert. Auf Basis einer geschätzten Zahl von 100-200 Kindern aus Familien mit hartem Drogenabusus ergibt sich eine Todeswahrscheinlichkeit von 2,3-4,6\% für die ersten 5 Lebensjahre. Die Wahrscheinlichkeit aller deutschen Kinder, in den ersten 5 Jahren $\mathrm{zu}$ versterben, beträgt rund $0,3 \%$ [14]. Bei elterlichem Drogenabusus ist diese Wahrscheinlichkeit somit 10-fach erhöht.

\section{Diskussion}

Mithilfe der vorliegenden Daten kann erstmalig systematisch und quantitativ vorgestellt werden, die den Handlungsempfehlungen der neuen Kinderschutzleitlinie [7] bereits weitgehend entsprechen bzw. teils sogar darüber hinausgehen.

\section{》) In Aachen werden bereits präpartal Hilfesysteme für Familien mit harten Drogenkonsum installiert}

Schon präpartal werden in der Stadt Aachen alle Mütter bzw. Paare zentral beim Jugendamt gemeldet, die bereits in der Schwangerschaft durch Drogenabusus auffällig werden bzw. in einem Substitutionsprogramm betreut sind. Hierbei ist eine enge Zusammenarbeit von meldenden Hausärzten, Sozialarbeitern, Substitutionspraxen, Gynäkologen, Hebammen und auch pränatal involvierten Kinderärzten notwendig, wobei jedoch auch Hinweise aus der Bevölkerung keine unerhebliche Rolle spielen. Erschwerend ist, dass gerade in suchtbelasteten Familien ein Schamund Schuldgefühl der betroffenen Eltern oder Angst vor Fremdunterbringung der Kinder dazu führen kann, dass Hilfesysteme nicht in Anspruch genommen werden und keine Überwachung der Schwangerschaft erfolgt. In der Stadt Aachen werden schon präpartal Hilfesysteme installiert, wie beispielsweise die Begleitung der drogenabhängigen Eltern durch Hebammen oder die Aufnahme der Mutter in eine Mutter-Kind-Einrichtung bereits 6 Wochen vor Geburt. Hierbei soll zum einen die werdende Mutter auf die Situation nach der Geburt vorbereitet werden, zum anderen besteht ein sog. Klärungsauftrag, der beinhaltet, die Ressourcen der Familie einzuschätzen und zu bewerten.

In der juristischen Betrachtung birgt die Problematik des Drogenkonsums während der Schwangerschaft verfassungs- und strafrechtliche Aspekte. So können sich die Rechte der Schwangeren und des Ungeborenen widersprechen, denn die Schwangere kann sich auf ihr allgemeines Persönlichkeitsrecht und das Recht auf körperliche Unversehrtheit beziehen, während dem Ungeborenen 
bereits der Schutz der Menschenwürde aus Artikel 1 des Grundgesetzes zusteht. Hieraus ergibt sich ein Spannungsfeld, in dem Hilfemaßnahmen eingegliedert werden müssen und staatliche Institutionen sowohl gesetzeskonform der Schwangeren gegenüber handeln, jedoch gleichzeitig die Rechte des Ungeborenen schützen müssen [16].

Ab der Geburt tritt die Rolle der Pädiater in den Vordergrund. Hierbei besteht die Aufgabe des betreuenden Kinderarztes zum einen in der Erkennung und Therapie von Kindern mit neonatalem Abstinenzsyndrom oder anderweitigen Auffälligkeiten durch präpartalen Drogenkonsum. Zum anderen kommt ihm die deutlich schwierigere Aufgabe zu, Mütter/Eltern mit bisher unbekanntem Drogenmissbrauch zu identifizieren, auch wenn zunächst keine offensichtlichen Beeinträchtigungen des Kindes vorliegen. Der Kinderarzt sollte trotz kurzer Kontaktzeiten im Rahmen von Vorsorgeuntersuchungen, Impfterminen oder Notfallvorstellungen ein Augenmerk auf derartige Anzeichen legen, um ggf. Unterstützung anbieten oder den Kontakt zu einem lokal verfügbaren Hilfesystem herstellen zu können.

\section{》) Ab der Geburt tritt die Rolle der Pädiater in den Vordergrund}

An dieser Stelle greift auch das Modellprojekt „Soziale Prävention in der Kinder- und Jugendarztpraxis" an, das durch das Land Nordrhein-Westfalen bis Dezember 2019 durchgeführt wurde. Hierbei wurden Kinderärzte in der ambulanten Praxis durch Mitarbeitende der Jugendhilfe unterstützt, indem regelmäßige Sprechzeiten in den Räumen der Kinder- und Jugendarztpraxis angeboten wurden. So konnten Familien unkompliziert und in bekannter Umgebung beraten und gleichzeitig die Familienstrukturen und das Risikopotenzial abgeschätzt werden. Somit handelt es sich um ein niedrigschwelliges, jedoch anscheinend effektives Angebot in der Kooperation von Kinderarzt und zuständigem Jugendamt [4].

Neben weiteren hier nicht aufgeführten stadtspezifischen Angeboten leistet v. a. das Netzwerk „Frühe Hilfen“ wichtige Arbeit in der Betreuung von Eltern und Kindern unter 3 Jahren und kooperiert dabei häufig mit dem Jugendamt. Insgesamt ist jedoch festzuhalten, dass es sich bei allen Maßnahmen um schwierige, ressourcenintensive Aufgaben handelt.

Betrachtet man nun die dargestellten Kasuistiken, waren die beschriebenen Kindeswohlgefährdungen im Voraus schwer erkennbar. In 2 Fällen (Kasuistiken 2 und 4) war der Drogenkonsum der Eltern erfolgreich verheimlicht worden; in 2 Fällen fanden unüberwachte Hausgeburten statt (Kasuistiken 3 und 5); bei Kasuistik 3 handelte es sich auch noch um eine Frühgeburt. In Kasuistik 1 nahm die Mutter einen kurzfristigen Ortswechsel vor, wobei die beteiligten Jugendämter tatsächlich miteinander Kontakt aufnahmen. Jedoch wurde die Situation sowohl durch das Jugendamt als auch durch die Substitutionspraxis nicht als akut bedrohlich für das Kind eingeschätzt. Insgesamt zeigen die dargestellten Fälle, dass elterlicher Abusus von harten, illegalen Drogen fatale Folgen für die im Haushalt lebenden Kinder haben kann. Hierbei erscheinen Verbesserungen bzw. Lösungsansätze zur Vermeidung solcher Situationen nicht trivial. Eine Primärprävention insbesondere von Ereignissen rund um den Geburtszeitpunkt gelingt unter den derzeitigen gesetzlichen und sozialen Bedingungen nicht immer. Es ist jedoch festzuhalten, dass insbesondere Hebammen, Gynäkologen und Personen aus dem Umkreis eine wichtige Rolle in der Früherkennung solcher Familien mit hartem Drogenkonsum zukommt. Intensivierte Schulungs- und Informationssysteme aller Beteiligten und eine breite Information der Öffentlichkeit wären möglicherweise hilfreich. Aus den Kasuistiken 2 und 3 könnte geschlussfolgert werden, dass alle Kinder von drogenabhängigen Eltern möglicherweise außerhäuslich untergebracht werden müssten. Hierzu sind weitere Untersuchungen mit größeren Fallzahlen und umfangreiche Diskussionen von Experten und dem Gesetzgeber erforderlich.

Zusammenfassend stellt der Konsum harter Drogen durch die Eltern einen zentralen Risikofaktor für eine akute Gefährdung der Kinder mit 10-fach erhöh- ter Mortalitätsrate dar. Damit gehört der Tod durch Drogenkonsum der Eltern zu einer der häufigsten Todesursachen in der Gruppe der 0- bis 5-Jährigen.

\section{Fazit für die Praxis}

Der Abusus harter Drogen der Eltern führt häufig zu einer relevanten Kindeswohlgefährdung, die sich in der vorliegenden Beobachtungsstudie als eine der führenden Todesursachen in der Altersklasse von 0 bis 5 Jahren herausstellte.

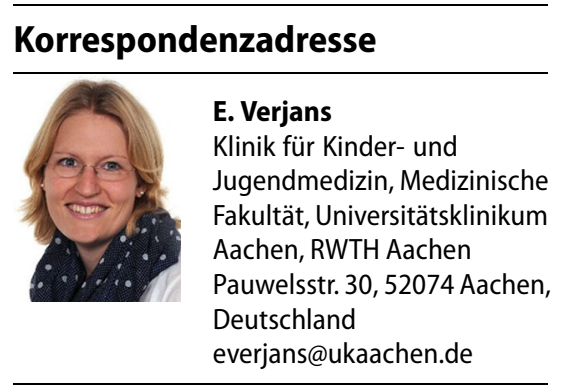

Funding. Open Access funding enabled and organized by Projekt DEAL.

\section{Einhaltung ethischer Richtlinien}

Interessenkonflikt. E. Verjans, U. Fabry und N. Wagner geben an, dass kein Interessenkonflikt besteht.

Diese retrospektive Studie erfolgte nach Konsultation der zuständigen Ethikkommission und im Einklang mit nationalem Recht.

Open Access. Dieser Artikel wird unter der Creative Commons Namensnennung 4.0 International Lizenz veröffentlicht, welche die Nutzung, Vervielfältigung, Bearbeitung, Verbreitung und Wiedergabe in jeglichem Medium und Format erlaubt, sofern Sie den/die ursprünglichen Autor(en) und die Quelle ordnungsgemäß nennen, einen Link zur Creative Commons Lizenz beifügen und angeben, ob Änderungen vorgenommen wurden.

Die in diesem Artikel enthaltenen Bilder und sonstiges Drittmaterial unterliegen ebenfalls der genannten Creative Commons Lizenz, sofern sich aus der Abbildungslegende nichts anderes ergibt. Sofern das betreffende Material nicht unter der genannten Creative Commons Lizenz steht und die betreffende Handlung nicht nach gesetzlichen Vorschriften erlaubt ist, ist für die oben aufgeführten Weiterverwendungen des Materials die Einwilligung des jeweiligen Rechteinhabers einzuholen.

Weitere Details zur Lizenz entnehmen Sie bitte der Lizenzinformation auf http://creativecommons.org/ licenses/by/4.0/deed.de. 


\section{Literatur}

1. Barber JG, Gilbertson R (1999) The drinker's children. Subst Use Misuse 34:383-402. https:// doi.org/10.3109/10826089909035652

2. Calhoun S, Conner E, Miller M, Messina N (2015) Improving the outcomes of children affected by parental substance abuse: a review of randomized controlled trials. Subst Abuse Rehabil 6:15-24. https://doi.org/10.2147/SAR.S46439

3. Conners-Burrow N, McKelvey L, Kyzer A et al (2013) Violence exposure as a predictor of internalizing and externalizing problems among children of substance abusers. J Pediatr Nurs 28:340-350. https://doi.org/10.1016/j.pedn.2012.11.006

4. Deutsche Gesellschaft für Kinder-und Jugendmedizin e. V. (DGKJ) (2020) Soziale Prävention in der Kinder-und Jugendarztpraxis. DGKJ, Berlin

5. Die Drogenbeauftragte der Bundesregierung (2017) Drogen- und Suchtbericht. Die Drogenbeauftragte der Bundesregierung, Berlin

6. Eickhorst A, Fullerton B (2017) Familiäre Belastungen von Eltern mit Kleinkindern im Überblick. Faktenblatt 2 zur Prävalenz- und Versorgungsstudie. Natl.Zent. Frühe Hilfen der Bundeszentrale für gesundheitliche Aufklärung

7. Kinderschutzleitlinie (2019) Kindesmisshandlung, -missbrauch, -vernachlässigung unter Einbindung der Jugendhilfe und Pädagogik. AWMF-Register 027-069

8. Klein M (2003) Kinder suchtkranker Eltern-Fakten, Risiken, Lösungen. In: Familiengeheimnisse wenn Eltern suchtkrank sind und die Kinder leiden. Bundesministerium für Gesundh. und Soz. Sicherung, Berlin

9. Kölch M, Ziegenhain U, Fegert J (2014) Kinder psychisch kranker Eltern - Herausforderungen für eine interdisziplinäreKooperation in Betreuung und Versorgung. Beltz, Basel

10. Leeb R (2008) Child maltreatment surveillance: uniform definitions for public health and recommended data elements. Centers for Disease Control and Prevention, National Center for Injury Prevention and Control, Atlanta

11. Moesgen D, Klein M, Dyba J (2017) Abhängigkeitserkrankungen und Elternschaft - Herausforderungen und Möglichkeiten der Hilfe. Suchttherapie 18:65-72. https://doi.org/10.1055/ s-0043-103060

12. Orwin RG, Maranda M, Ellis B (2000) The effectiveness of substance abuse treatment in reducing violent behavior. J Psychopathol Behav Assess 22:309-324. https://doi.org/10.1023/A: 1007687622994

13. Rounsaville D, O'Farrell TJ, Andreas JB et al (2014) Children's exposure to parental conflict after father's treatment for alcoholism. Addict Behav 39:1168-1171. https://doi.org/10.1016/j.addbeh. 2014.03.017

14. United Nations Inter-agency group for Child Mortality Estimation (UN IGME) (2019) Levels and trends in child mortality: report 2019. UN IGME, New York

15. Velleman R, Templeton LJ (2016) Impact of parents' substance misuse on children: an update. BJPsych Adv 22:108-117. https://doi.org/10.1192/apt.bp. 114.014449

16. Wissenschaftlicher Dienst des Bundestages (2020) Zum Schutz des Ungeborenen bei einer Drogenoder Alkoholsucht der Schwangeren (WD 9-3000093/19) 